\title{
Hydroelastic waves on fluid sheets
}

\author{
M. G. Blyth ${ }^{1} \dagger$, E. I. Părău ${ }^{1}$ and J.-M. Vanden-Broeck ${ }^{2}$ \\ ${ }^{1}$ School of Mathematics, University of East Anglia, Norwich NR4 7TJ, UK \\ 2 Department of Mathematics, University College London, London WC1E 6BT, UK
}

(Received 28 July 2011; revised 6 October 2011; accepted 13 October 2011; first published online 9 November 2011)

Nonlinear travelling waves on a two-dimensional inviscid fluid sheet are investigated when the sheet is bounded above and below by two thin elastic plates. Symmetric and antisymmetric solution branches are identified, together with a pair of bifurcation branches. It is shown that far along the branches the solutions approach limiting configurations that correspond to static solutions valid in the absence of fluid forcing.

Key words: elastic waves, waves/free-surface flows

\section{Introduction}

The interaction between a moving fluid and a deformable boundary is a topic of fundamental interest in fluid mechanics (e.g. Korobkin, Părău \& Vanden-Broeck 2011). Theoretical understanding of hydroelasticity can be applied to numerous industrial problems and it aids understanding of a host of naturally occurring phenomena. In this paper we discuss two-dimensional hydroelastic waves propagating on sheets of fluid that are bounded by elastic plates. The fluid motion is assumed to be both inviscid and irrotational. Two elastic plates sandwich a layer of moving fluid and deform according to the dynamic pressure exerted by the fluid. The plates are assumed to be thin and upon deformation to develop both in-plane and transverse shear tensions, as well as a bending moment. The fluid motion and elastic deformation are coupled together via a dynamic balance relating the hydrodynamic loading to the elastic stresses prevailing in the plates.

In the case when the transverse shear tension and bending moment are both identically zero, the in-plane tension is constant and the more classical problem of capillary waves travelling along fluid sheets is recovered. In this case Taylor (1959) noted that there are two types of small-amplitude waves: symmetric and antisymmetric. Nonlinear solutions were calculated exactly by Kinnersley (1976). Crowdy (1999) gave a simpler method of computing the same waves using a complex variable technique. New solutions that bifurcate from the main symmetric Kinnersley branch were identified by Blyth \& Vanden-Broeck (2004). In the present problem we find that again only symmetric and antisymmetric waves exist at small amplitude, and we trace the nonlinear development of the waves as an appropriate elasticity parameter is varied for a fixed flux of fluid through the sheet.

Although the present study is purely theoretical, practical applications in which hydroelastic waves occur are manifest. Examples include flapping flags (e.g. Shelley,

$\dagger$ Email address for correspondence: m.blyth@uea.ac.uk 
Vandenberghe \& Zhang 2005) and biomedical applications such as heart valves (e.g. Jia et al. 2007) and air flow in the windpipe (e.g. Grotberg 1994). In particular, our work may find application in flat-plate-type fuel assemblies found in nuclear reactor cooling systems. These assemblies are composed of a number of elastic plates placed in parallel to each other, with coolant fluid flowing through the gaps between them (e.g. Kim \& Davis 1995). In this application it is desired that the plates remain flat and in parallel during operation. However, when the coolant flow rates between the plates are large, the plates may deform.

In $\S 2$ we formulate the parallel elastic plate problem to be studied. In $\S 3$ we consider static solutions in the absence of a fluid motion, and in $\S 4$ we study travelling waves when there is a prevailing flow between the plates.

\section{Problem formulation}

We investigate periodic waves of wavelength $\lambda$ propagating at constant speed along a fluid sheet confined between two elastic plates as illustrated in figure 1. Gravity is neglected, and the fluid motion is assumed to be inviscid and both incompressible and irrotational. The plates are free to deform to accommodate the travelling waves. As the plates deform, they develop both in-plane and shear tensions, which are balanced by the pressure in the liquid. The regions above the upper plate and beneath the lower plate are assumed to be dynamically inactive and at a constant pressure, which is taken to be zero.

Disregarding inertia, a mechanical equilibrium balance of forces and moments in the elastic plates yields (e.g. Flaherty, Keller \& Rubinow 1972)

$$
\gamma^{\prime}+\kappa q=0, \quad q^{\prime}-\kappa \gamma= \pm p, \quad q=m^{\prime},
$$

where a prime denotes differentiation with respect to arclength $s$ along the plate, $\gamma(s)$ and $q(s)$ are the tangential and shear tensions in the plate, respectively, with units of force per unit length, $m(s)$ is the bending moment, and $p$ is the pressure in the liquid. The plus/minus sign applies at the upper/lower plate respectively. The curvature $\kappa=\theta^{\prime}(s)$, where $\theta$ is the angle made between the tangent to an elastic plate and the $x$ axis. For the bending moment, we adopt the simple linear relationship suggested by previous workers,

$$
m=E_{B} \kappa,
$$

where $E_{B}$ is a bending modulus. Thin-shell theory predicts that $E_{B}=E h^{3} /\left[12\left(1-v^{2}\right)\right]$, where $E$ is Young's modulus, $v$ is Poisson's ratio and $h$ is the plate thickness, which is here assumed to be constant (e.g. Fung 1965). The relationship (2.2) has been justified for the case of an inextensible elastic plate, as assumed here, by Pozrikidis (2002). Integrating (2.1) once with respect to arclength and eliminating $\gamma$ and $q$, we obtain the dynamic relationship between the pressure and elastic stresses,

$$
p= \pm E_{B}\left(\kappa^{\prime \prime}+\frac{1}{2} \kappa^{3}-\sigma \kappa / \lambda^{2}\right),
$$

where $\sigma=\lambda^{2} \gamma / E_{B}+\lambda^{2} \kappa^{2} / 2$ is a constant of integration. The plus sign applies at the upper plate and the minus sign applies at the lower plate. We remark that (2.3) is consistent with the recent hydroelastic formulation of Plotnikov \& Toland (2011).

We work in a frame of reference $(x, y)$ moving at the constant speed of the wave, as shown in figure 1. The wave speed of the elastic plate is assumed to be the same as that of the liquid - note that the formulation of Plotnikov \& Toland (2011) allows for different wave speeds. To define the wave speed, $c$, we take the average velocity over 


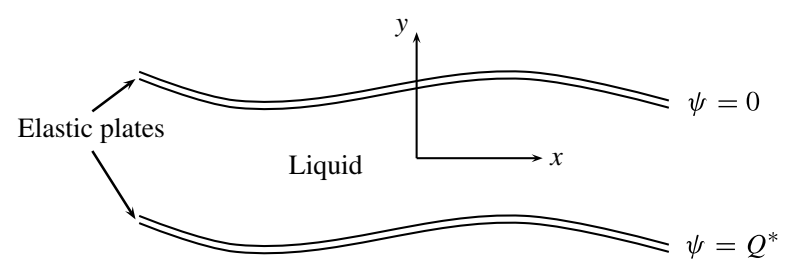

FIGURE 1. Sketch of periodic waves travelling along a liquid sheet bounded by two elastic plates. Arclength $s$ is measured as increasing from left to right on both of the elastic plates.

one period of a streamline, writing

$$
c=\frac{1}{\lambda} \int_{x=0}^{x=\lambda} \boldsymbol{u} \cdot \mathrm{d} \boldsymbol{x}
$$

where $\mathrm{d} \boldsymbol{x}=(\mathrm{d} x, \mathrm{~d} y)$ is parallel to a streamline, $\boldsymbol{u}=(u, v)=\nabla \phi$ is the fluid velocity and $\phi(x, y)$ is the velocity potential. Since the flow is irrotational, $c$ is the same for any choice of streamline. It follows that, when $x$ changes by $\lambda$, then $\phi$ changes by $c \lambda$.

Since the fluid motion inside the sheet is governed by Laplace's equation, it is useful to introduce a complex potential $f=\phi+\mathrm{i} \psi$, where $\psi(x, y)$ is the stream function defined so that $\psi=0$ on the upper surface and $\psi=Q^{*}<0$ on the lower surface. Since $\phi$ and $\psi$ are both harmonic functions, $f$ is analytic and it only remains to satisfy the normal stress balance at the interfaces between the liquid and the elastic plates. Applying Bernoulli's equation at the interfaces and using (2.3) we obtain

$$
\frac{1}{2} U^{2} \pm \frac{E_{B}}{\rho}\left(\kappa^{\prime \prime}+\frac{1}{2} \kappa^{3}-\frac{\sigma \kappa}{\lambda^{2}}\right)=c^{2} B
$$

where the plus/minus signs follow the same convention as above, $\rho$ is the density of the liquid and $U=|\boldsymbol{u}|$ is the fluid speed at the respective plate. The Bernoulli constant $c^{2} B$ is assumed to be the same on both plates and is to be found. Before proceeding, we non-dimensionalize using $c$ as the reference velocity and $\lambda$ as the reference length, and define the dimensionless flux $Q=Q^{*} /(c \lambda)<0$. Henceforth all quantities presented are dimensionless.

We seek solutions periodic in $\phi$ with unit period. All of the waves to be computed are assumed to be symmetric about $\phi=0$. In order to compute the waves we use essentially the same method as that adopted by Blyth \& Vanden-Broeck (2004). We introduce the analytic function $\tau(\phi, \psi)-\mathrm{i} \theta(\phi, \psi)$, which is defined so that

$$
u-\mathrm{i} v=\mathrm{e}^{\tau-\mathrm{i} \theta} .
$$

In non-dimensional form, (2.5) becomes

$$
\frac{1}{2} U^{2} \pm D\left(\kappa^{\prime \prime}+\frac{1}{2} \kappa^{3}-\sigma \kappa\right)=B
$$

where $U=\mathrm{e}^{\tau}$ and the curvature terms are given by

$$
\kappa=\mathrm{e}^{\tau} \frac{\partial \theta}{\partial \phi}, \quad \kappa^{\prime \prime}=\mathrm{e}^{3 \tau}\left[2\left(\frac{\partial \tau}{\partial \phi}\right)^{2} \frac{\partial \theta}{\partial \phi}+3 \frac{\partial \tau}{\partial \phi} \frac{\partial^{2} \theta}{\partial \phi^{2}}+\frac{\partial^{2} \tau}{\partial \phi^{2}} \frac{\partial \theta}{\partial \phi}+\frac{\partial^{3} \theta}{\partial \phi^{3}}\right]
$$

with the dimensionless parameter $D$ defined by

$$
D=\frac{E_{B}}{\rho c^{2} \lambda^{3}} .
$$


We recall that the plus/minus signs apply to the upper/lower plate, respectively. The dimensionless constant $\sigma$ is arbitrary and can be thought of as a measure of prestressing of the elastic plates prior to deformation. In the simplest case of a liquid sheet of uniform thickness with parallel elastic plates, the curvature is zero and we obtain $\sigma=D^{-1}\left(\gamma / \rho c^{2} \lambda\right)$, where the term in the bracket is the dimensionless tension in the plates; if the plates are unstressed, this tension is zero and so $\sigma=0$.

One may infer from the linear stability theory of De Langre (2002) that two types of periodic wave are possible when the deformation on the elastic plates is small, namely symmetric waves, where a trough on the upper wave is above a peak on the lower wave, and antisymmetric waves, where a trough on the upper wave is above a trough on the lower wave, as shown in figure 1. Assuming waves of small amplitude, our preceding analysis leads to the dispersion relations

$$
\operatorname{coth}(\pi Q)+2 \pi D\left(\sigma+4 \pi^{2}\right)=0, \quad \tanh (\pi Q)+2 \pi D\left(\sigma+4 \pi^{2}\right)=0,
$$

for symmetric and antisymmetric waves, respectively. Our goal is to compute largeamplitude waves for different values of the governing parameters, namely $Q, D$ and $\sigma$. The linear dispersion relations for small-amplitude capillary waves are obtained from (2.10) in the limit $D \rightarrow 0$ while holding the product $D \sigma$ fixed. The waves will be calculated using a numerical method to be described in $\S 4$. First we present a short discussion of static solutions in the absence of fluid motion. It will be seen later that these are useful for interpreting the travelling wave solutions in an appropriate limit.

\section{Static solutions}

When there is no flow in the sheet, the fluid pressure is constant and there is no mechanical coupling between the two elastic plates. The balance of forces in the upper plate, for example, demands that the term in the bracket on the left-hand side of (2.7) vanish. Integrating once with respect to arclength we obtain

$$
\kappa^{\prime 2}+\frac{1}{4} \kappa^{4}-\sigma \kappa^{2}=d,
$$

where $d$ is a constant of integration. It is possible to integrate (3.1) to obtain $\kappa(s)$ in terms of elliptic functions. However, this leads to rather complicated formulae (see Vassilev, Djondjorov \& Mladenov 2008) and so, as an alternative and more convenient approach, we compute solutions numerically. Before attempting a numerical solution, it is helpful first to examine the possible solution trajectories in the $\left(\kappa, \kappa^{\prime}\right)$ phase plane. Two representative trajectories are shown in figure 2(a) for $\sigma=0$ and $\sigma=0.5$, both for the sample choice $d=1$. The large dots indicate the points $\left(-k_{0}, 0\right)$ and $\left(k_{0}, 0\right)$. In the case $\sigma=0$ we have $k_{0}=(4 d)^{1 / 4}$.

The qualitative shape of the orbits shown in figure 2(a) indicates the existence of periodic solutions for $\kappa$. To compute the periodic wave profiles, we rewrite (3.1) as a first-order system and integrate numerically using the fourth-order Runge-Kutta method starting from the left-hand dot and advancing to the righthand dot (see figure $2 a$ ) to complete the upper half of an orbit. The lower half is completed by symmetry. The profile of the plate in physical $(x, y)$ space is obtained by simultaneously integrating the system $x^{\prime \prime}=-\kappa y^{\prime}, y^{\prime \prime}=\kappa x^{\prime}$, where the prime denotes differentiation with respect to arclength. Consistent with the current nondimensionalization, we further demand that $x$ changes by a unit amount as we travel around the circuit in figure $2(a)$. So, if $S$ represents the total arc distance covered in travelling through a complete orbit in the phase plane, then defining $x(S) \equiv X$, we require that $X=1$. 

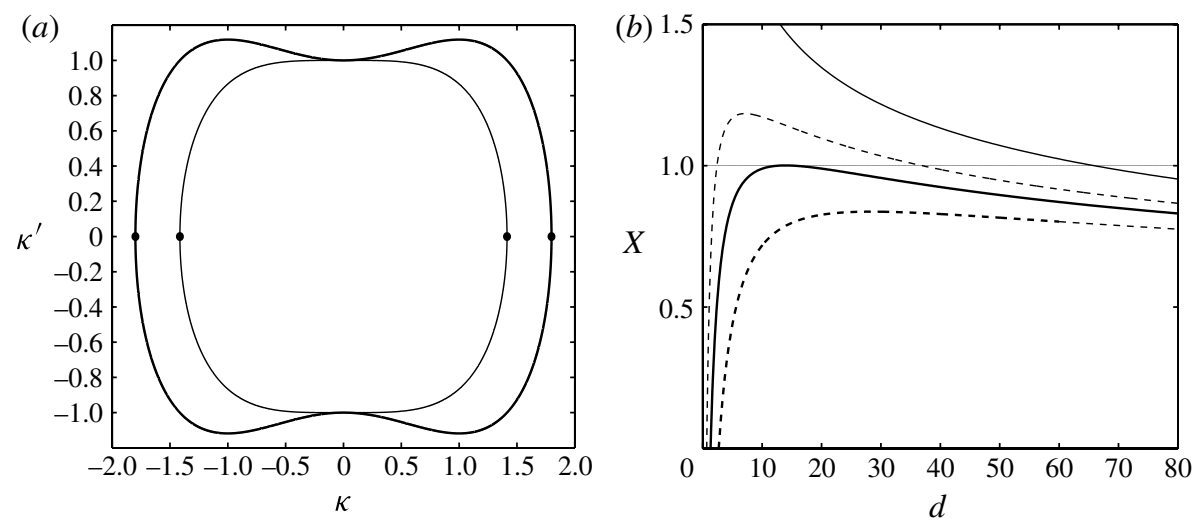

FIGURE 2. (a) Typical phase portraits of (3.1) in the $\left(\kappa, \kappa^{\prime}\right)$ plane: $\sigma=0, d=1$, thin line; $\sigma=0.5, d=1$, thick line. (b) Variation of $X$ with $d$ for solutions of (3.1) for various $\sigma: \sigma=0$, thin solid; $\sigma=0.6$, thin dashed; $\sigma=1.4$, thick solid; and $\sigma=2.0$, thick dashed. Also shown is a thin line indicating the level $X=1$.
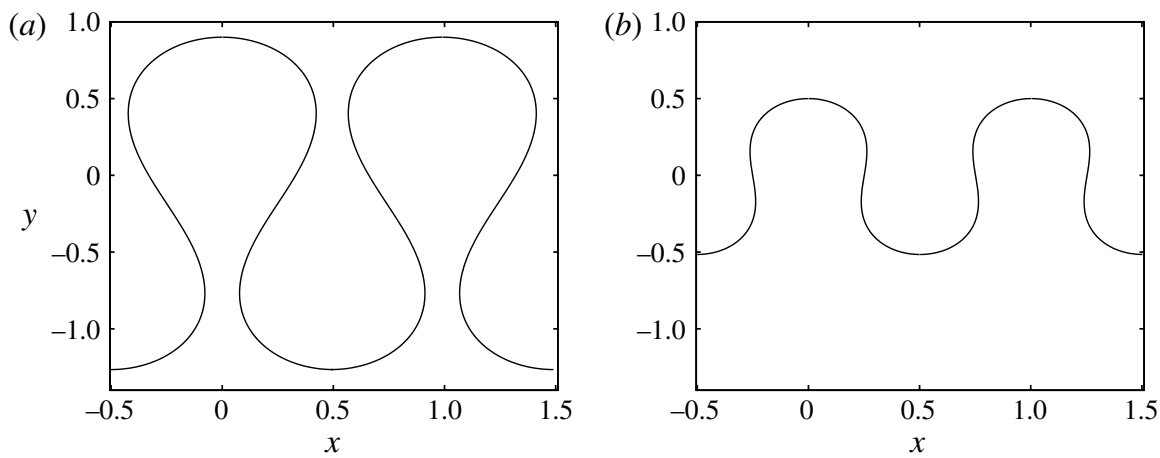

FIGURE 3. Static wave profiles for $\sigma=1.0$ : $(a) d=2.4$ and $(b) d=36.9$.

The condition $X=1$ can be satisfied by an appropriate choice of the integration constant $d$. In figure $2(b)$ we show how $X$ varies with $d$ for a number of choices of $\sigma$. When $\sigma=0$ we find that $X$ approaches infinity as $d \rightarrow 0$. For $\sigma>0$, as $d$ decreases, $X$ attains a maximum value before reaching zero at a finite value of $d$. For $\sigma=0$ there is one candidate value of $d$, for $0<\sigma<1.4$ there are two, and for $\sigma>1.4$ there are none. Sample wave profiles are shown in figure 3 for the case $\sigma=1.0$ when $d=2.4$ and $d=36.9$ are the two possibilities. As $\sigma$ is decreased, the profile corresponding to the smaller value of $d$ eventually undergoes self-intersection.

Profiles featuring $n$ waves in one period of unit length, where $n \in \mathbb{Z}$, may be obtained by seeking $d$ such that $X=1 / n$. For a further discussion of periodic solutions to (3.1) see Vassilev et al. (2008).

\section{Travelling wave solutions}

We compute travelling wave solutions to (2.7) numerically using a collocation method. Following Vanden-Broeck \& Miloh (1995) we express the solution in the 
form

$$
\tau-\mathrm{i} \theta=a_{0}+\sum_{n=1}^{\infty} a_{n} \mathrm{e}^{2 \mathrm{i} n \pi f}+\sum_{n=1}^{\infty} b_{n} \mathrm{e}^{-2 \mathrm{i} n \pi f},
$$

where $a_{0}$ and the coefficients $a_{n}$ and $b_{n}$ are to be found and are all real. The latter follows from the assumed symmetry about $\phi=0$. In numerical practice we truncate each of the series in (4.1) after a finite number of terms $N-1$. Next we introduce the $N$ collocation points $\phi_{j}^{(u)}$ on the upper plate, where $\psi=0$, and the $N-1$ points $\phi_{j}^{(l)}$ on the lower plate, where $\psi=Q$ :

$$
\phi_{j}^{(u)}=\frac{j-1}{2 N}, \quad j=1, \ldots, N ; \quad \phi_{j}^{(l)}=\frac{j-1}{2(N-1)}, \quad j=1, \ldots, N-1 .
$$

We then apply (2.7) with the plus sign at the $N$ upper collocation points and (2.7) with the minus sign at the $N-1$ lower collocation points. Notice that we only collocate one half of each wave. Next, in keeping with the non-dimensionalization above, we demand that $x$ varies by a unit amount over one wavelength. Equivalently, because of the wave symmetry,

$$
\int_{0}^{1 / 2} \frac{\partial x}{\partial \phi} \mathrm{d} \phi=\int_{0}^{1 / 2} \mathrm{e}^{-\tau} \cos \theta \mathrm{d} \phi=\frac{1}{2} .
$$

We apply (4.3) on the upper plate only, and discretize the integral using the trapezium rule with $N$ intervals defined by the collocation points (4.2). We now have a total of $2 N$ nonlinear algebraic equations for the $2 N$ unknowns including $a_{n}(n=0, \ldots, N-1)$, $b_{n}(n=1, \ldots, N-1)$ and the Bernoulli constant $B$. We solve the equations for a chosen value of $D$ using Newton's method with a suitable initial guess. Alternatively, it may be more convenient to fix the value of $a_{1}$ and to compute $D$ as part of the solution. Once a solution has been computed, $x$ and $y$ are obtained by integrating the identity

$$
\frac{\partial x}{\partial \phi}+\mathrm{i} \frac{\partial y}{\partial \phi}=\frac{1}{u-\mathrm{i} v}=\mathrm{e}^{-\tau+\mathrm{i} \theta} .
$$

The wave separation at a chosen $\phi$ is computed by evaluating numerically the integral

$$
\int_{Q}^{0} \frac{\partial y}{\partial \psi} \mathrm{d} \psi=\int_{Q}^{0} \mathrm{e}^{-\tau} \cos \theta \mathrm{d} \psi
$$

To provide a check on the numerical method, we compared the results against the linear dispersion relations (2.10) and confirmed excellent agreement. In practice, when computing large-amplitude waves we find that a large value of $N$ is required to obtain an accurate solution. For example, for some of the solutions to be presented in the next section, a value of $N=800$ or more is required, leading to very long computation times. To reduce the size of $N$ while maintaining the same level of accuracy, we can impose the following relations between the coefficients: $a_{n}=\mathrm{e}^{2 \pi n Q} b_{n}$ for symmetric waves, and $a_{n}=(-1)^{n} \mathrm{e}^{2 \pi n Q} b_{n}$ for antisymmetric waves (see Blyth \& Vanden-Broeck 2004 for an explanation of these relations). The value of $N$ used in each case is quoted in the figure captions. 

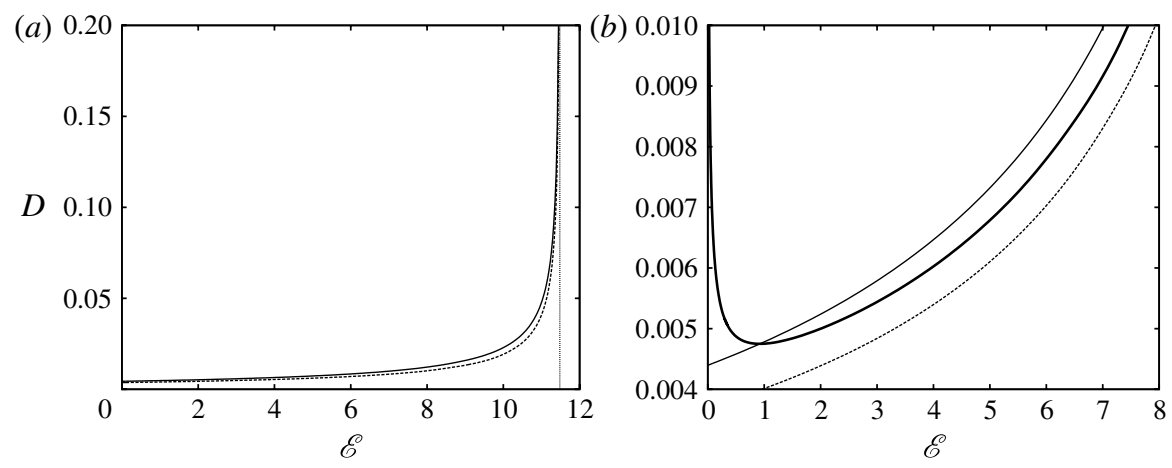

Figure 4. $Q=-0.5, \sigma=0$. (a) The symmetric (solid line) and antisymmetric (broken line) solution branches. The asymptote shown as a dotted line at $\mathscr{E}=11.48$ corresponds to the limiting static configuration. (b) The bifurcation branches (thick line) emanating from the symmetric branch (solid line). The antisymmetric branch is shown as a broken line.

\subsection{Results and discussion}

We characterize the solutions using the dimensionless measure of elastic bending energy,

$$
\mathscr{E}=\frac{\lambda}{2} \int_{0}^{L} \kappa^{2} \mathrm{~d} s=\frac{1}{2} \int_{0}^{1} \mathrm{e}^{\tau}\left(\frac{\partial \theta}{\partial \phi}\right)^{2} \mathrm{~d} \phi
$$

where $L$ is the total arclength of the deformed plate in one period and $\kappa$ is the curvature in one or other of the plates. The main focus of the ensuing discussion is on the case $Q=-0.5$ and $\sigma=0$; other values are considered towards the end of the section.

Small-amplitude waves are symmetric or antisymmetric, obey the appropriate dispersion relation in (2.10), and are sinusoidal in profile. As $D$ increases, the waves tend to increase in amplitude and nonlinear effects come into play. Figure 4(a) shows the solution space for the case $Q=-0.5$ and $\sigma=0$ on a graph of $D$ against $\mathscr{E}$. The solid line is the symmetric solution on which wave profiles are symmetric. The broken line is the antisymmetric solution branch. These two branches connect with the predictions of linear theory as $\mathscr{E} \rightarrow 0$. Two other solution branches, which bifurcate nonlinearly from the symmetric solution branch, are shown in figure $4(b)$ with thick lines.

Sample wave profiles along the symmetric solution branch are plotted in figure 5 . Typical wave profiles along the antisymmetric branch are shown in figure 6. As $D$ increases, the bending energy $\mathscr{E}$ approaches an asymptotic limit, as can be seen in figure $4(a)$, and the wave profiles approach a limiting configuration. A wave profile on the symmetric branch that is close to the limiting profile is shown in figure 7(a) for $D=2.0$. Only one half of a wavelength of the lower wave is shown. When $D$ is increased from this value, the profile changes very slightly but not significantly.

Referring to (2.7), it is reasonable to suppose that for large $D$ the solution may be approximated to leading order by setting the bracketed terms in that equation to zero. This is tantamount to seeking a static solution as discussed in $\S 3$. The wave profile obtained by solving the static plate (3.1) for $\sigma=0$ is shown as a broken line in figure $7(a)$. The agreement between this and the limiting travelling wave profile is striking. The static profile corresponds to the point where the solid line in figure $2(b)$ 

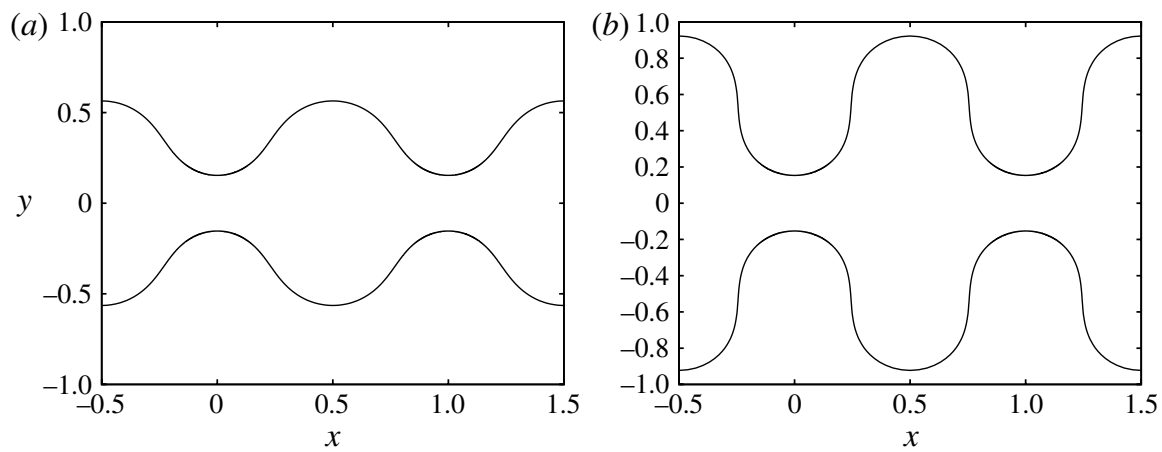

FigURE 5. $Q=-0.5, \sigma=0$. Wave profiles on the symmetric branch for $(a) D=0.0133$ and (b) $D=0.2$. The waves were computed with $N=800$. Wave profiles on the symmetric branch for $D>0.2$ are very similar to those shown in $(b)$.
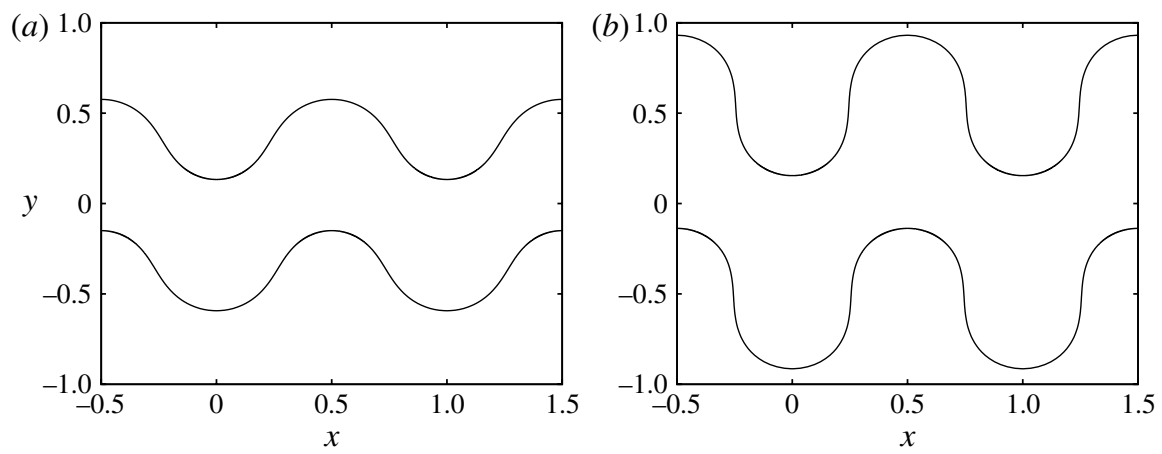

FIGURE 6. $Q=-0.5, \sigma=0$. Wave profiles on the antisymmetric branch for (a) $D=$ 0.0133 and $(b) D=0.2$. The waves were computed with $N=800$. Wave profiles on the antisymmetric branch for $D>0.2$ are very similar to those shown in $(b)$.
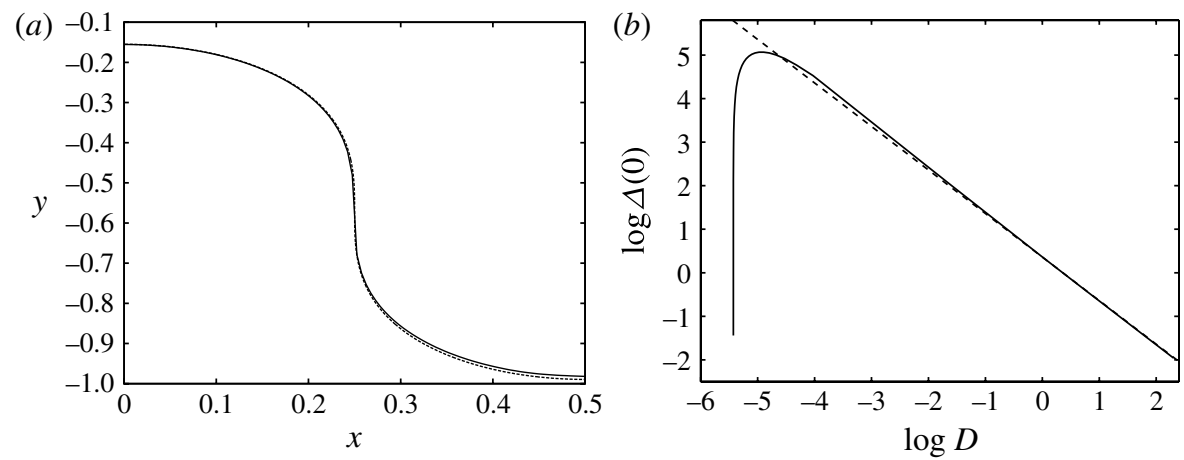

FIgURE 7. $Q=-0.5, \sigma=0$. (a) A near-limiting lower wave profile (solid line) on the symmetric branch at $D=2.0(N=800)$. One half of one wavelength is shown. The barely visible broken line is the solution of the static plate equation (3.1). (b) Plot of $\log \Delta(0)$ versus $\log D$. The broken line is of slope -1 and intercepts the horizontal axis at $\log (1.4)$. 

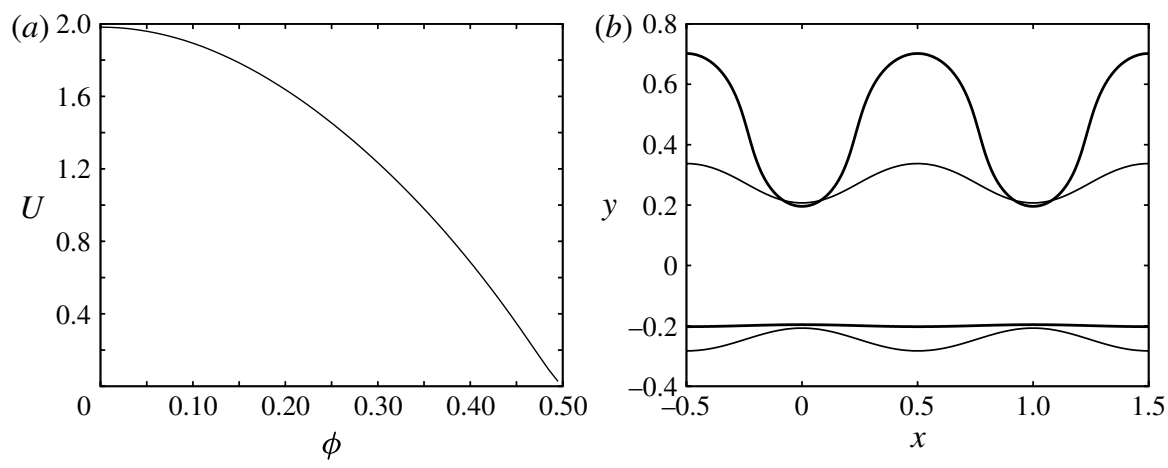

Figure 8. $Q=-0.5, \sigma=0$. (a) The velocity $U$ along the lower wave plotted against $\phi$. (b) Wave profiles on the lower bifurcation branch for $D=0.0048(N=60$, thin lines) and $D=0.02(N=240$, thick lines).

meets the level $X=1$. The energy for this static profile is $\mathscr{E}=11.48$, and it is towards this value, shown with a dotted line in figure $4(a)$, that the energies on the symmetric and antisymmetric branches asymptote as $D$ becomes large.

To provide further evidence that the wave profiles are indeed attaining the static profiles in the limit $D \rightarrow \infty$, we examine the size of the modulus of the terms in brackets in (2.7) for large values of $D$. We define $\Delta(\phi) \equiv\left|\kappa^{\prime \prime}+\kappa^{3} / 2-\sigma \kappa\right|$ and consider the variation of $\log \Delta(0)$ with $\log D$ as $D$ increases. (Note that $\Delta(0)$ is the maximum value of $\Delta$ over one wavelength.) This relationship is plotted in figure 7(b) for the wave profiles on the symmetric branch for the case $\sigma=0$. The plot indicates that $\Delta \approx 1.4 / D$ when $D$ is sufficiently large. The factor of 1.4 has been obtained by fitting a straight line to the data, shown as a broken line in the figure. In general, this factor will depend on $Q$. We have also confirmed numerically that $\Delta$ is inversely proportional to $D$ for $D$ large on the antisymmetric branch.

Although the limiting profile coincides with the static solution, it is important to note that this does not mean that the fluid inside the sheet is not moving, merely that the elastic forces in the plate dominate over the dynamic pressure imposed by the fluid. This becomes clear, for example, by looking at the velocity, $U$, along one wavelength of the wave, and this is shown in figure 8(a) for the lower wave. The velocity approaches zero at $\phi=0.5$, which is at the mid-point of the lower wave trough (the rightmost point on the wave shown in figure $7 a$ ). Noting the comments of the previous paragraph, we see that consistency is maintained in (2.7). Since the bracketed terms decay like $D^{-1}$, we obtain a balance across all terms in the equation at the next order of approximation.

The bifurcation branches were discovered by examining the determinant of the Jacobian matrix used for Newton's method when solving the system of nonlinear algebraic equations formulated in $\S 2$. In common with the problem of pure capillary waves studied by Blyth \& Vanden-Broeck (2004), we found that there are no bifurcations along the antisymmetric solution branch. Wave profiles along the lower bifurcation branch are plotted in figure $8(b)$. A limiting state is reached far along the branch in which the lower wave profile becomes flat and the upper wave approaches a static wave solution. Wave profiles along the upper bifurcation branch are shown in figure $9(a)$. In this case it is the upper wave profile that flattens out far along the bifurcation branch. 

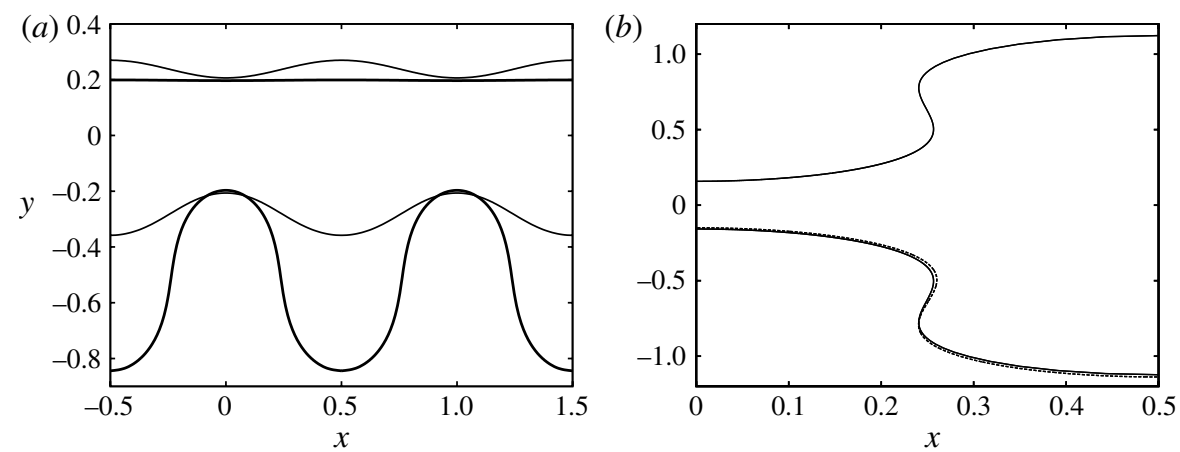

Figure 9. $Q=-0.5$. (a) Profiles on the upper bifurcation branch for $D=0.005(N=80$, thin lines) and $D=0.05$ ( $N=200$, thick lines). (b) An overturning profile for $\sigma=0.9$ $(N=1900)$. The broken line overlaid onto the lower wave is the static wave prediction for $d=40.3$.

The static solutions computed in $\S 3$ suggest that limiting travelling wave configurations featuring overturning should be attained if $\sigma$ is taken to be large enough. In figure $9(b)$ we show the computed upper and lower wave profiles for the case $\sigma=0.9$. Superimposed onto the lower wave is the static wave prediction for the larger of the two possible values of $d$ (recall from the discussion in $\S 3$ that, for some values of $\sigma$, of which $\sigma=0.9$ is included, there are two possible static profiles; further comment on this is made below).

This discussion has focused on the value $Q=-0.5$. The results are similar for other values of $Q$. For all the cases that we examined, we found only one bifurcation point along the symmetric branch and none on the antisymmetric branch. As $Q$ increases, the bifurcation point on the symmetric branch moves to smaller values of $D$. This behaviour contrasts with the situation for capillary waves, where the number of bifurcations along the symmetric branch varies with $Q$ (Blyth \& Vanden-Broeck 2004).

For pure capillary waves, the elastic terms (corresponding to the first two terms in brackets in (2.7)) are absent. Large-amplitude solutions have been discussed by Kinnersley (1976), who computed exact solutions, and by Blyth \& Vanden-Broeck (2004), who discovered some new bifurcation branches. Our results reduce to those for pure capillary waves in the limit $D \rightarrow 0$ while holding $D \sigma$ fixed, which effectively removes the elastic terms from (2.7). Solutions that are close to those seen for pure capillary waves may be computed within the present framework by selecting a small value of $D$ and a moderate value of $D \sigma$. We have computed such solutions and found that they look very similar to those appearing in figure 2(d) of Blyth \& Vanden-Broeck (2004) so that the waves are almost self-touching.

Our results for static plates suggest that, when $\sigma$ lies within certain bounds, there are three possible profiles: a flat plate and two non-trivial solutions with large-amplitude deformation. We have demonstrated that our travelling wave solutions approach one of these static solutions in the limit of large $D$. However, there remains the issue of whether or not the other non-trivial static solution is relevant to the dynamics of the liquid sheet. It might be hypothesized that there exists another travelling wave solution branch that approaches the other non-trivial static solution in some limit. We have not been able to identify such a branch and we leave its possible existence as an open question. 


\section{Acknowledgements}

This work was supported in part for J.M.V.-B. by EPSRC under grant $\mathrm{EP} / \mathrm{H} 022740 / 1$, and for E.I.P. by EPSRC under grant EP/H008489/1.

\section{REFERENCES}

Blyth, M. G. \& VAnden-Broeck, J. M. 2004 New solutions for capillary waves on fluid sheets. J. Fluid Mech. 507, 255-264.

Crowdy, D. G. 1999 Exact solutions for steady capillary waves on a fluid annulus. J. Nonlinear Sci. 9, 615-640.

De LAngre, E. 2002 Absolutely unstable waves in inviscid hydroelastic systems. J. Sound Vib. 256 (2), 299-317.

Flaherty, J. E., Keller, J. B. \& Rubinow, S. I. 1972 Post buckling behaviour of elastic tubes and rings with opposite sides in contact. SIAM J. Appl. Math. 23 (4), 446-455.

FunG, Y. 1965 Foundations of Solid Mechanics. Prentice-Hall.

Grotberg, JB 1994 Pulmonary flow and transport phenomena. Annu. Rev. Fluid Mech. 26 (1), 529-571.

JiA, L. B., LI, F., YIN, X. Z. \& YIN, X. Y. 2007 Coupling modes between two flapping filaments. J. Fluid Mech. 581 (1), 199-220.

KIM, G. \& DAVIS, D. C. 1995 Hydrodynamic instabilities in flat-plate-type fuel assemblies. Nucl. Engng Des. 158 (1), 1-17.

KinNersley, W. 1976 Exact large amplitude waves on sheets of fluid. J. Fluid Mech. 77, 229-241.

Korobkin, A., PărăU, E. I. \& VAnden-Broeck, J. M. 2011 The mathematical challenges and modelling of hydroelasticity. Phil. Trans. R. Soc. Lond. A 369, 2803-2812.

Plotnikov, P. I. \& Toland, J. F. 2011 Modelling nonlinear hydroelastic waves. Phil. Trans. $R$. Soc. Lond. A 369, 2942-2956.

POZRIKIDIS, C. 2002 Buckling and collapse of open and closed cylindrical shells. J. Engng Maths 42 (2), 157-180.

Shelley, M., Vandenberghe, N. \& Zhang, J. 2005 Heavy flags undergo spontaneous oscillations in flowing water. Phys. Rev. Lett. 94 (9), 94302.

TAYLOR, G. I. 1959 The dynamics of thin sheets of fluid. II. Waves on fluid sheets. Proc. R. Soc. Lond. A. 253, 296-312.

VAnden-Broeck, J. M. \& Miloh, T. 1995 Computations of steep gravity waves by a refinement of Davies-Tulin's approximation. SIAM J. Appl. Maths. 55 (4), 892-903.

Vassilev, V. M., Djondjorov, P. A. \& Mladenov, I. M. 2008 Cylindrical equilibrium shapes of fluid membranes. J. Phys. A 41, 435201. 